\title{
BET bromodomain inhibitors synergize with ATR inhibitors in melanoma
}

\author{
This article has been corrected since Online Publication and an Erratum has also been Published
}

\author{
Somsundar Veppil Muralidharan ${ }^{1}$, Berglind Osk Einarsdottir ${ }^{1}$, Joydeep Bhadury ${ }^{1,2}$, Mattias F Lindberg ${ }^{1}$, Jin Wu ${ }^{3}$, Eric Campeau ${ }^{3}$, \\ Roger Olofsson Bagge ${ }^{1}$, Ulrika Stierner ${ }^{4}$, Lars $\mathrm{Ny}^{4}$, Lisa M Nilsson ${ }^{1}$ and Jonas A Nilsson ${ }^{\star, 1}$
}

\begin{abstract}
Metastatic malignant melanoma continues to be a challenging disease despite clinical translation of the comprehensive understanding of driver mutations and how melanoma cells evade immune attack. In Myc-driven lymphoma, efficacy of epigenetic inhibitors of the bromodomain and extra-terminal domain (BET) family of bromodomain proteins can be enhanced by combination therapy with inhibitors of the DNA damage response kinase ATR. Whether this combination is active in solid malignancies like melanoma, and how it relates to immune therapy, has not previously investigated. To test efficacy and molecular consequences of combination therapies cultured melanoma cells were used. To assess tumor responses to therapies in vivo we use patient-derived xenografts and B6 mice transplanted with B16F10 melanoma cells. Concomitant inhibition of BET proteins and ATR of cultured melanoma cells resulted in similar effects as recently shown in lymphoma, such as induction of apoptosis and p62, implicated in autophagy, senescence-associated secretory pathway and ER stress. In vivo, apoptosis and suppression of subcutaneous growth of patient-derived melanoma and B16F10 cells were observed. Our data suggest that ATRI/BETI combination therapies are effective in melanoma.
\end{abstract}

Cell Death and Disease (2017) 8, e2982; doi:10.1038/cddis.2017.383; published online 10 August 2017

Malignant melanoma (MM) is potentially curable if diagnosed early but if the disease becomes metastatic it often is fatal. Recent advances in the molecular and immunological characterization of the disease have generated new promising avenues of therapeutic intervention. ${ }^{1}$ First, the discovery of the mutated BRAF oncogene ${ }^{2}$ enabled the development of targeted kinase inhibitors that exhibited remarkable objective response in patients with $\mathrm{MM}$ carrying the mutated $B R A F^{V 600}$ allele. ${ }^{3,4}$ Additional inhibition of the downstream MAPK pathway using MEK inhibitors has further prolonged overall survival ${ }^{5}$ but in most cases relapses of lethal and therapy resistant clones emerge. Multiple resistance pathways have been found, ${ }^{6,7}$ suggesting new combination treatments that are tested in various clinical trials. Second, the discovery of immune checkpoints ${ }^{8}$ enabled the development of antibodies directed against CTLA4 and PD-1 (or its ligand PDL1), which show lower response rates but generally more durable responses. ${ }^{9,10}$ Taken together, these modern treatments have been successful, but to cure or make MM manageable chronic, more and safe drugs are needed.

BET proteins are emerging targets for cancer therapy. ${ }^{11}$ BET proteins regulate transcription and appear to be utilized during cancer progression to epigenetically reprogram both blood and solid cancers. ${ }^{12}$ Small-molecule inhibitors of BET proteins are in clinical trials but preclinical models already suggest that combination therapies will be needed to further the efficacy of BET inhibitors (BETIs). Indeed, we and other investigators have recently demonstrated that inhibitors targeting cell-signaling molecules, the proteasome, components of the DNA damage response and HDAC synergize with BETIs to kill B-cell malignancies. ${ }^{13-19}$ HDAC and BETI combination treatment is also effective in melanoma ${ }^{20}$ but whether any of the other therapies would be effective is not known.

Ataxia-telangiectasia and Rad3-related (ATR) is a kinase that belongs to the PI3-kinase-like family, which also includes PI3K, mTOR, ATM and DNA-PK. ATR has a critical role in the regulation of replication and is activated by replication fork stalling. Known causes of stalling include UV-induced DNA damage and nucleotide deprivation but also excess replication fork firing exerted by oncogenic replication stress. ${ }^{21}$ When ATR is activated it phosphorylates the checkpoint kinase Chk1 that work together with ATR to phosphorylate components in the replication machinery to inhibit further replication. Hence, inhibition of ATR or Chk1 is detrimental to cells experiencing replication fork problems such as cancer cells expressing high levels of the MYC oncogene. ${ }^{22-25}$

Both BETls and Chk1 inhibitors have previously been shown to have efficacy in cultured melanoma cells and Chk1 has even been suggested to be essential for the melanocytic lineage. $^{26}$ We have demonstrated that Myc-induced lymphoma cells undergoing replication stress, because of ATR inhibition, are sensitive to BETIs. ${ }^{19}$ Here we wish to investigate whether or not this finding can be extended to solid cancers. By using cultured melanoma cells, patient-derived xenografts (PDXs) and syngenic transplant models we show that the

\footnotetext{
${ }^{1}$ Sahlgrenska Cancer Center, Department of Surgery or University Hospital, Gothenburg, Sweden; ${ }^{2}$ The Institute of Medical Science, Division of Stem Cell Therapy, The University of Tokyo, Tokyo, Japan; ${ }^{3}$ Zenith Epigenetics Ltd, Calgary, Canada and ${ }^{4}$ Department of Oncology, Institute of Clinical Sciences, Sahlgrenska Academy, University of Gothenburg and The Sahlgrenska University Hospital, Gothenburg, Sweden

${ }^{*}$ Corresponding author: JA Nilsson, Department of Surgery, Sahlgrenska Cancer Center, Medicinaregatan 1G, Plan 6, Gothenburg 41390, Sweden. Tel: +46 31 7866768; E-mail: jonas.a.nilsson@ surgery.gu.se

Received 22.2.17; revised 25.6.17; accepted 02.7.17; Edited by R Aqeilan
} 
a

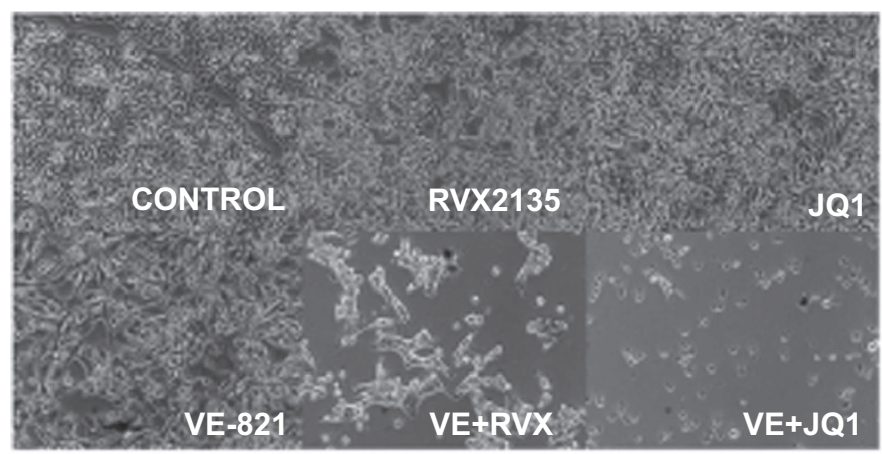

MeWo

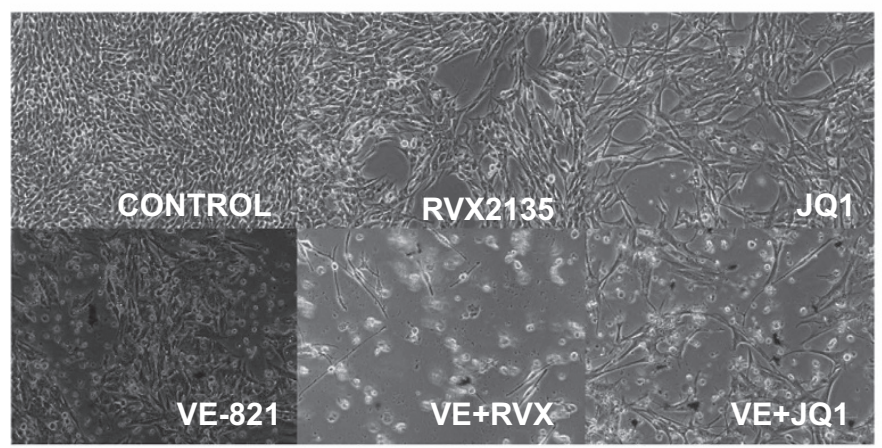

C
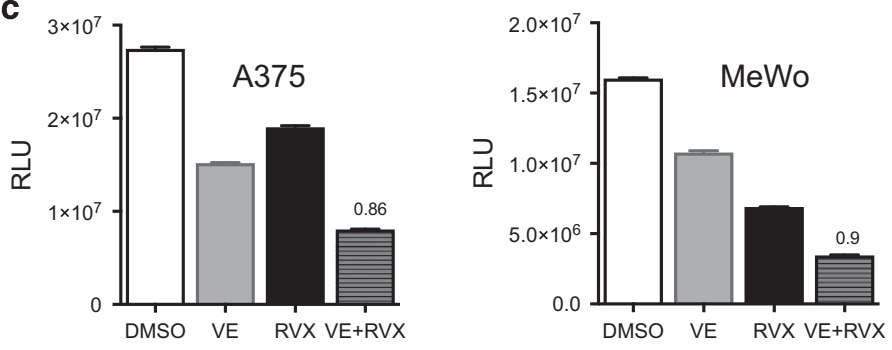
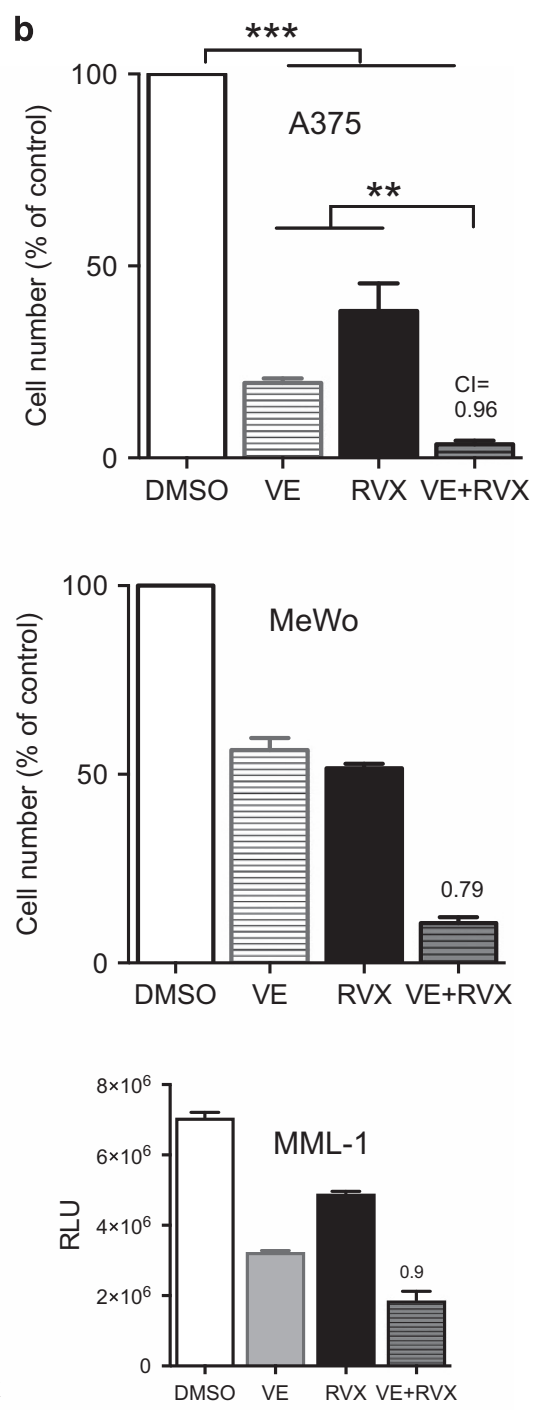

d

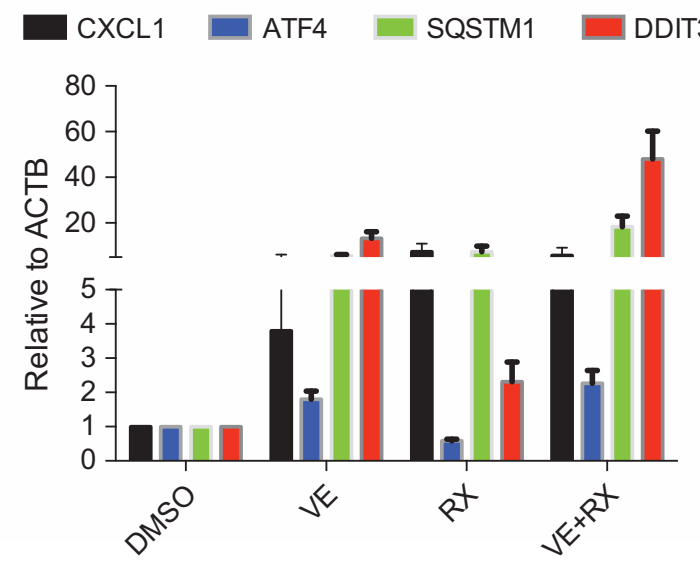

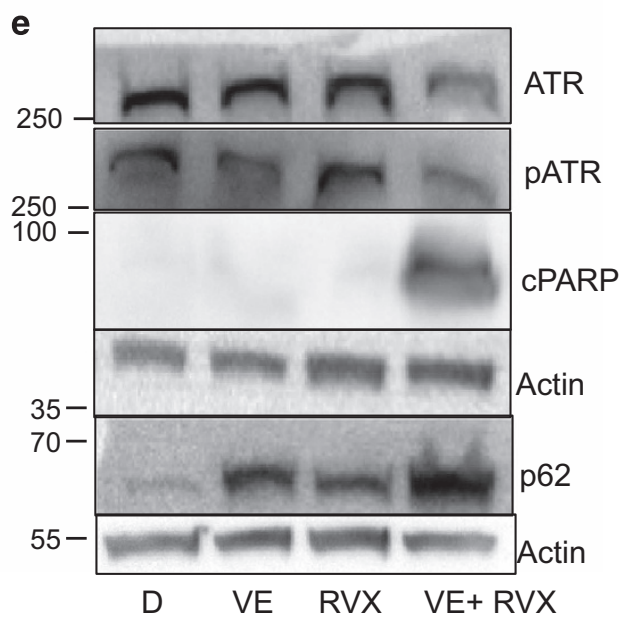

Figure 1 ATRIs synergize with BETi to kill melanoma cells and induce SASP/ER stress. (a and $\mathbf{b}) \mathrm{A} 375 \mathrm{cells}\left(B R A F^{\mathrm{V} 600 \mathrm{E}}\right)$ and MeWo cells $\left(N F 1^{-1-}\right)$ were treated with vehicle (0.1\% DMSO), $10 \mu \mathrm{M}$ VE821 (VE; AXON Medchem, Groningen, The Netherlands), $1 \mu \mathrm{M}$ JQ1 (Cayman Chemicals, Ann Arbor, MI, USA), $10 \mu \mathrm{M}$ RVX2135 (RVX) or indicated combinations. The experiments were repeated twice in biological triplicates. Cells were imaged in a light microscope (a) or counted in a hemocytometer (b). (c) A375 and MeWo cells were cultured in the presence of vehicle (DMSO), $10 \mu \mathrm{M}$ of RVX2135 (RVX) and/or the ATRI VE821 (VE; $10 \mu \mathrm{M}$ ) for $48 \mathrm{~h}$, and were assayed for viability with CellTiter Glo. Value to achieve synergy is shown with a dotted line. (d) A375 cells treated with vehicle, $10 \mu \mathrm{M} \mathrm{VE821,} 10 \mu \mathrm{M}$ RVX2135 or both VE821/RVX2135 were analyzed by qRT-PCR for indicated genes. (e) A375 cells treated as described above and analyzed by western blot analysis using indicated antibodies 
therapeutic combination targeting of ATR and BET proteins is effective in melanoma.

\section{Results and Discussion}

BET bromodomain inhibitors synergize with ATR inhibitors to induce apoptosis, and senescence-associated secretory pathway in melanoma. Melanoma cells are sensitive to the BETIS JQ1, iBET-151 and RVX2135 (Supplementary Figure S1 and shown by others ${ }^{15,27,28}$ ). To assess the therapeutic effect of combined inhibition of ATR kinase and BET protein we cultured the melanoma cell lines A375 and MeWo in the presence of the ATR inhibitor (ATRI) VE821 and/or RVX2135. ${ }^{15,19}$ Both compounds were antiproliferative as assessed by microscopy, CellTiter Glo (Promega, Madison, WI, USA) measurements and cell counts (Figures $1 \mathrm{a}-\mathrm{c}$ ). Combining the two generated profound effects on the viability of the cells and combination index calculations showed that the compounds synergized (Figures $1 b$ and $c$ ).

In our previous study we showed that ATRI/BETI combination therapy of B-cell lymphoma resulted in a gene expression profile resembling that of senescence-associated secretory pathway (SASP) and ER stress. ${ }^{19}$ Examining the melanoma cells treated with ATRIs/BETls that had not undergone apoptosis it was evident that the cells had large vacuoles or vesicles in their cytoplasm; this was mostly evident in combination-treated cells but also seen in ATRI-treated cells (Figure 1a). We therefore performed qRT-PCR and western blot analyses on A375 cells and probed for components of SASP/ER stress that we had found deregulated in lymphoma cells responding to the combination treatment. Indeed, the mRNA encoding the SASP cytokine Cxcl1, the ER stress master regulator ATF4 and the SASP/ER stress regulators SQSTM/p62 and DDIT3/CHOP were all induced by ATR and in combination-treated cells albeit not in a synergistic manner
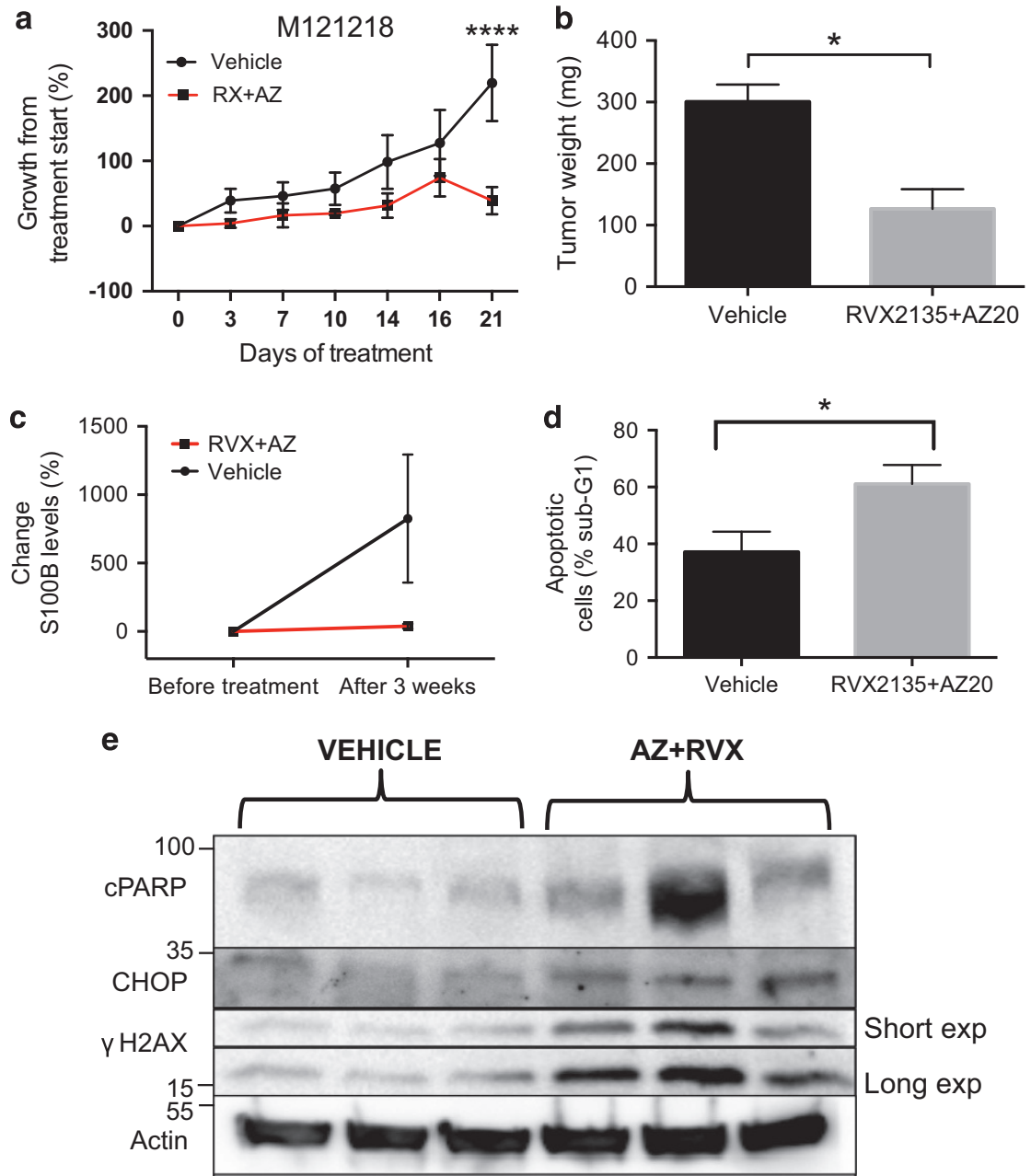

Figure 2 ATRIs synergize with BETi to kill melanoma cells. (a) The melanoma PDX model M121218 was initiated by thawing a stock of cryopreserved melanoma tumor cells, ${ }^{30}$ and injecting the cells subcutaneously into the flank of 10 immunocompromised NOD/SCID/IL2R $\gamma$ mice (Taconic). Tumor sizes were measured bi-weekly using an caliper. When the tumors reached $75-100 \mathrm{~mm}^{3} 5$ mice each were randomized to receive either oral and i.p. vehicle, or oral RVX2135 at $75 \mathrm{mg} / \mathrm{kg}$ b.i.d. and i.p. injection of AZ20 (MedChemExpress, Princeton, NJ, USA) at $50 \mathrm{mg} / \mathrm{kg}$ q.d. for 5 days a week. (b) Four hours after the last dose, tumors were excised and weighed. (c) A blood sample was drawn from the saphenous vein of all mice before treatment and after 3 weeks of treatment. Plasma was isolated and used to determine the level of the melanoma marker S100B using an ELISA kit from Abcam (Elisa kit from Abnova, Taipei City, Taiwan). (d) Single cells were derived by trypsinization of excised tumors from vehicle-treated or combination-treated mice. The cells were lysed and their nuclei were labeled with 7-AAD. Sub-G1 content (apoptosis) was measured by flow cytometry. (e) Tumor pieces from M121218 PDXs treated with vehicle or the RVX2135/AZ20 combination treatment were subjected to western blot analysis 

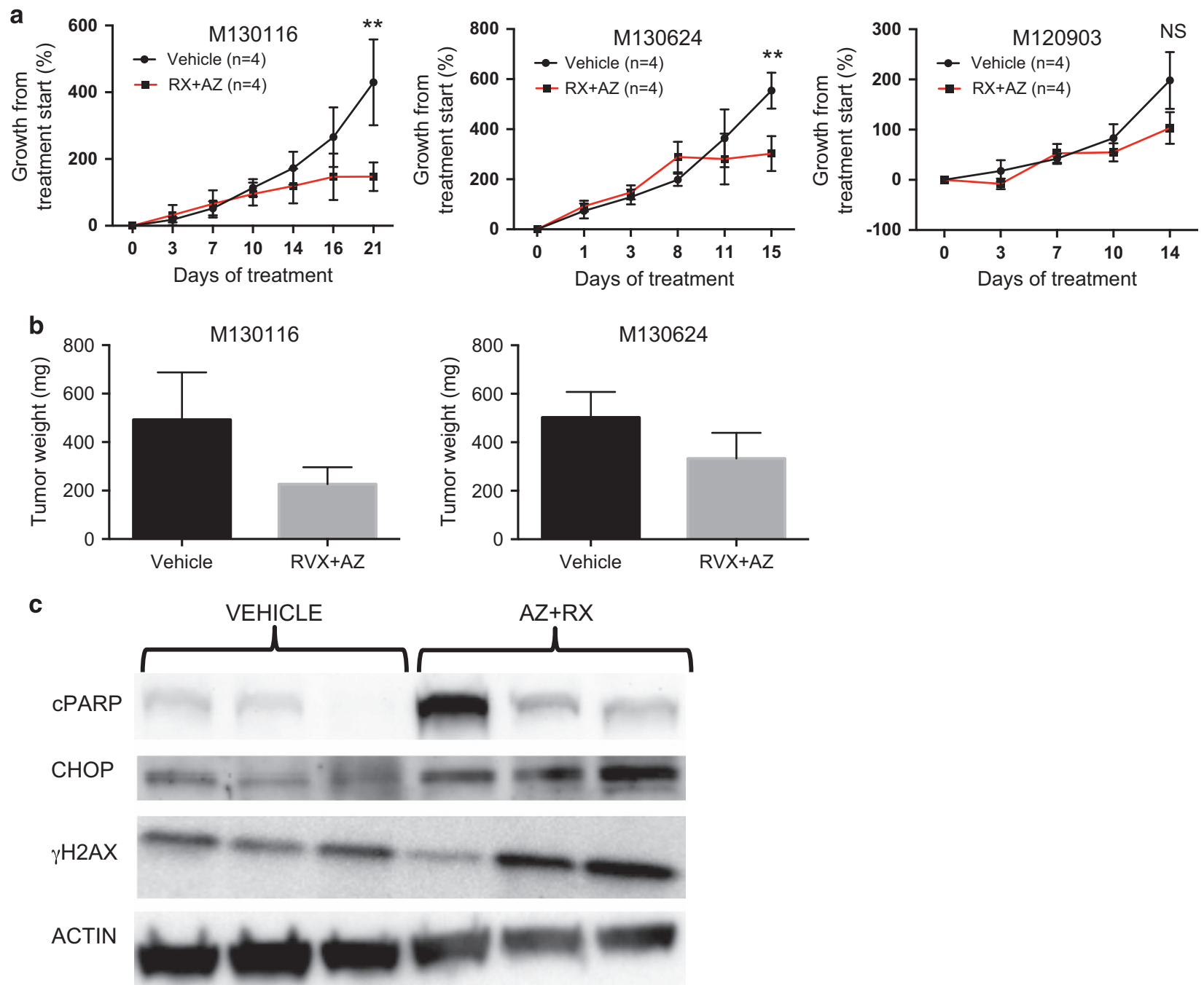

Figure 3 Combined ATR and BET inhibition reduces growth of patient-derived melanoma tumorgrafts in mice. (a) Growth of three melanoma PDX models, originally developed from a biopsies of patients' metastases, transplanted subcutaneously onto the flank of NOG mice ( $n=4$ per treatment group). When the tumors reached $75-100 \mathrm{~mm}^{3}$ they were randomized to receive either oral vehicle or $75 \mathrm{mg} / \mathrm{kg} \mathrm{RVX2135} \mathrm{b.i.d.} \mathrm{and} \mathrm{an} \mathrm{intraperitoneal} \mathrm{injection} \mathrm{of} \mathrm{vehicle} \mathrm{or} 50 \mathrm{mg} / \mathrm{kg}$ AZ20 q.d. five times per week. After the first week of treatment PDX model M120903 exhibited sings of treatment-induced distress so the dose of AZ20 was reduced to 25 mg/kg q.d. for all mice of all PDX models subsequently treated to avoid complications. (b) Four hours after the last dose, tumors were excised and weighed from the indicated PDX models. The tumor texture of M120903 was very loose preventing accurate weighing. (c) Tumor pieces from M130116 PDXs treated with the vehicle or the RVX2135/AZ20 combination treatment were subjected to western blot analysis

(Figure 1d). Western blot analysis confirmed that the combination treatment synergistically induced p62 (Figure 1e; Supplementary Figures S2A-C). The apoptotic marker cleaved PARP was induced in A375 cells and MeWo cells (Figure 1e), but Bim and the ER stress regulators CHOP and ATF4 (Supplementary Figures S2C and D) were not. As the ER stress inducer tunicamycin potently induced CHOP and ATF4 (Supplementary Figure S2C) it is possible that BETi/ATRi change the rate of translation or induce protein turnover, which would explain the discordance between the RNA and protein levels of CHOP and ATF4. Interestingly, GATA4, a component of SASP was downregulated (Supplementary Figure S2D), which could explain why a full SASP phenotype was absent. Taken together our data suggest that cultured melanoma cells are sensitive to ATRI/BETI combination treatment and hence that this new treatment is effective in more settings than Myc-induced lymphoma. ${ }^{19}$
ATRI and BET combination treatment can induce apoptosis, SASP and ER stress in melanoma tumors in mice. Cultured melanoma cells are grown in very different conditions than melanoma cells in patients or in mice. ${ }^{29}$ We recently developed a platform of highly characterized PDX models. ${ }^{30}$ To test whether ATRI/BETI would work in a PDX model end we had to use the bioavailable ATRI AZ20, as VE821 is not bioavailable in vivo. In PDX model M121218 we observed a robust reduction in subcutaneous growth and tumor size (Figures 2a and b), a reduced serum level of the melanoma marker S100B (Figure 2c), and a marked increase of apoptotic cells in the excised tumor (Figure 2d). To investigate whether apoptosis and SASP/ER stress was induced by ATRI/BETI treatment also in vivo we subjected excised tumors to western blot analysis (Figure 2e). As seen in vitro and in lymphoma ${ }^{19}$ there was an induction of cleaved 
PARP, indicating apoptosis, increased levels of SASP/ER stress marker DDIT3/CHOP and increased levels of phosphorylated $\mathrm{H} 2 \mathrm{Ax}(\gamma \mathrm{H} 2 \mathrm{AX})$, a marker of double-stranded DNA damage that often follows ATR/Chk1 inhibition. ${ }^{31,32}$

To investigate whether tumors from other patients would be sensitive to the ATRI/BETI combination therapy we treated three other PDX models. In two of these models models, the combination treatment blocked growth resulting in smaller tumors and induction of apoptosis (Figures $3 a-c$ ). In the fourth model, derived from a lymph node metastasis of patient M120903, the initiation of treatment resulted in adverse effects and drug-related death and the need to decrease the dose of AZ20 (Figure 3a). This is suggestive of tumor lysis syndrome akin to what was observed in lymphoma-bearing mice with large tumors treated with the ATRI/BETI combination treatment. $^{19}$

Finally, to test the effect of the combination in a mouse model, which has immune cells we turned to a widely used murine melanoma cell line, B16F10, which can be grown in syngenic $\mathrm{C} 57 \mathrm{BL} / 6$ mice. We first cultured B16F10 cells in vitro in the presence or absence of ATRI (VE821 or AZ20) and/or BETI (RVX2135 or iBET762). The cells were noticeably sensitive to $B E T I$, less to ATRI but very sensitive to the combination therapy (Figures $4 a-d$ ), irrespective of which BETI or ATRI that was used, suggesting on-target effects. Again vacuole-like or lysosome-like structures were evident in the combination-treated cells (Figure 4a), and long-term culture killed the cells, whereas single-treated cells were growth-inhibited (Figures $4 b-d$ ). We tested the effect of the ATRI/BETI treatment in vivo, by injecting luciferase-expressing B16F10 cells subcutaneously. One week after transplant, mice were imaged and then treatment was commenced. Because the single drugs were insufficient in vitro we tested the combination treatment in vivo. Reassuringly, treatment with the combination treatment reduced the luciferase signal from the B16F10 (Figure 4e). Taken together our data suggest that melanoma cells from humans and mice are sensitive to ATRI/BETI combination treatment in vitro and in vivo and hence that this new treatment could be effective in more settings than Myc-induced lymphoma. a

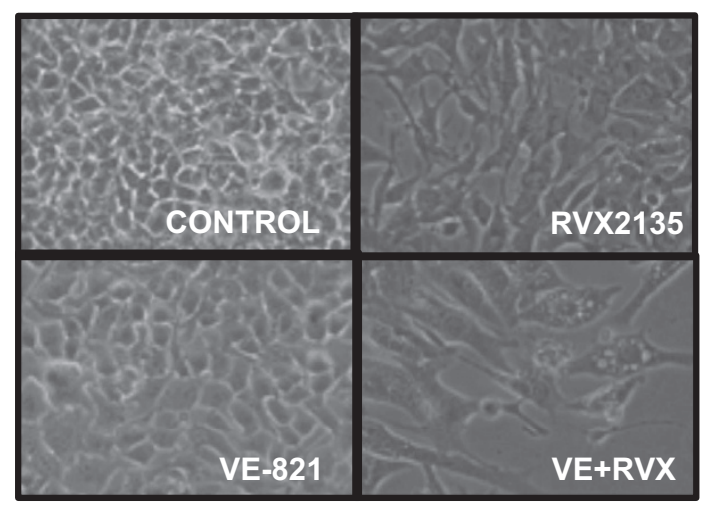

b

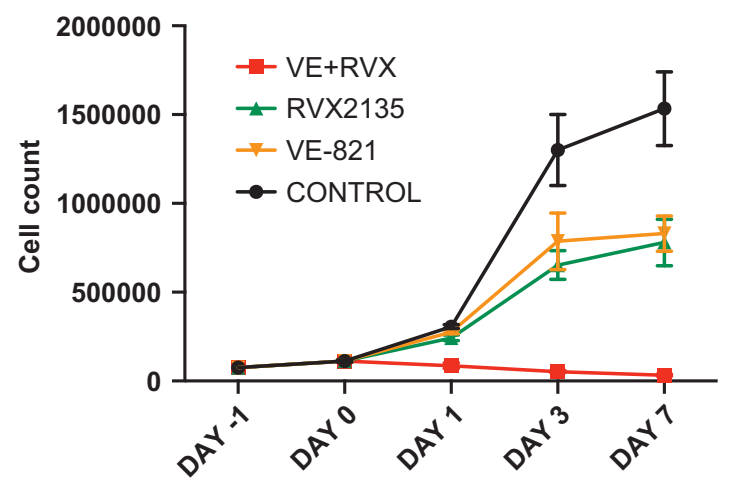

C

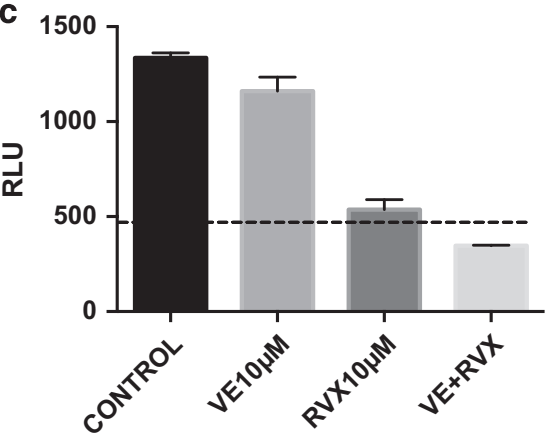

d

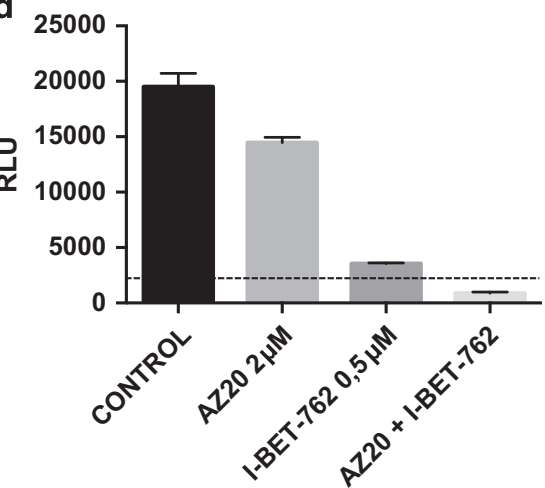

e

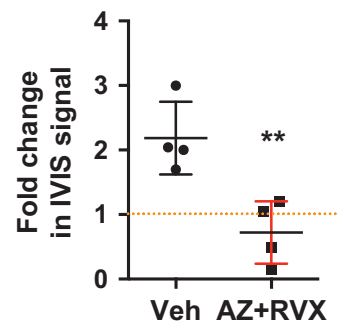

Figure 4 ATRI/BETI combination treatment kills B16 melanoma cells in vitro and in vivo. (a) B16F10-luciferase cells were cultured in RPMI-1640 supplemented with $10 \%$ fetal bovine serum and antibiotics. They were treated with vehicle (0.1\% DMSO), $10 \mu \mathrm{M}$ VE821 (VE), $10 \mu \mathrm{M}$ RVX2135 (RVX) or indicated combinations. The experiments were repeated twice in biological triplicates. Cells were imaged in a light microscope (a) or counted in a hemocytometer (b). (c) B16F10-luciferase cells were cultured in the presence of vehicle (DMSO), $10 \mu \mathrm{M}$ of RVX2135 (RVX) and/or the ATRI VE821 (VE; $10 \mu \mathrm{M}$ ) for $48 \mathrm{~h}$ and were assayed for viability by adding luciferin (Perkin-Elmer) to a final concentration of $100 \mu \mathrm{g} / \mathrm{ml}$. Value to achieve synergy is shown with a dotted line. (d) B16F10-luciferase cells were cultured in the presence of vehicle (DMSO), $0.5 \mu \mathrm{M}$ of iBET762 and/or $2 \mu \mathrm{M}$ of the ATRI AZ20 for $48 \mathrm{~h}$ and were assayed for viability by adding luciferin (Perkin-Elmer) to a final concentration of $100 \mu \mathrm{g} / \mathrm{ml}$. Value to achieve synergy is shown with a dotted line. (e) Eight 6- to 8-week-old C57BL/6 Albino mice were transplanted subcutaneously with $10^{5}$ B16F10-luciferase cells. Seven days after transplantation, mice were imaged in an IVIS Lumina III XR machine. After imaging, mice were treated with oral and i.p. vehicle, or oral RVX2135 at $75 \mathrm{mg} / \mathrm{kg}$ b.i.d. and i.p. injection of AZ20 at $25 \mathrm{mg} / \mathrm{kg}$ for 4 days, followed by imaging again. Shown is the fold change in luciferase signal during treatment ( $n=4$ mice per treatment group) 
To conclude, it is worth noticing that targeted therapies directed against the mutated driver BRAF have potent yet short-term effects and do not work in the half-of-all melanomas that lack BRAF V600 mutations. Immune therapies have longer effects but fewer patients respond. Therefore, additional therapies targeting the cancer cell's engine, rather than its driver, is needed. First, an obvious approach is to target the transcription factors deregulated in the cancers, such as MYC. The concept has been validated in many preclinical models ${ }^{33}$ but to date no effective therapy is present in the clinic. Second, other interesting targets are those harnessing the genetic stability - a known cancer cell vulnerability. ${ }^{34}$ Inhibitors of DNA repair proteins, such as PARP, ${ }^{35}$ and checkpoint kinases, such as Chk1 and ATR, ${ }^{23,25}$ are undergoing clinical development alone or in combination with classical chemotherapy. Third, to target epigenetic readers, writers and erasers is an additional approach capitalizing on the epigenetic changes in cancer cells that have been recognized for decades. Three HDAC inhibitors (HDACl) and two DNA methyltransferase inhibitors are already approved for various malignancies. Several BETI, histone/lysine methyltransferase inhibitors and HAT inhibitors are in various stages of development. Combination therapies are an obvious approach but which therapies to combine, when to combine and how to combine to maximize efficacy and minimize side effects is still unknown. We have identified two different possible combination therapies against Mycinduced cancers, BETI/HDACl ${ }^{15}$ and BETI/ATRI (here and Muralidharan et al. $^{19}$ ). It appears as if the mechanisms are broad, resulting in cell death and large changes in transcriptional output. On the other hand, it remains to be found whether or not there are selective events that are shared between these two potent combination therapies that can be further utilized in design of new therapeutic approaches.

\section{Materials and Methods \\ Cell experiments. All cell lines were from Cell Lines Services (Eppelheim, Germany). They were maintained in complete medium (RPMI-1640 supplemented with $10 \% \mathrm{FBS}$, glutamine and gentamycin) and cultured at $37^{\circ} \mathrm{C}$ with $5 \% \mathrm{CO}_{2}$. Viability following inhibitor treatment was monitored with CellTiter Glo.}

RNA and protein expression. RNA preparation is carried out using the Nucleospin RNA isolation kit (Macherey-Nagel, Düren, Germany). Following cDNA synthesis using iScript cDNA synthesis kit (Bio-Rad, Hercules, CA, USA) indicated genes were amplified using a SYBR green PCR mastermix (Kapa Biosystems, Woburn, MA, USA). Primer sequences are available on request. The $\Delta \Delta C T$ method was used to calculate the relative expression.

For western blot analysis of protein expression, cell pellets or tumor pieces lysed in lysis buffer as described before. ${ }^{25} \mathrm{~A}$ unit of $50 \mu \mathrm{g}$ of protein was resolved on $4-20 \%$ ClearPAGE gels (C.B.S Scientific Company, San Diego, CA, USA) and transferred to nitrocellulose membrane (Protran, GE Healthcare Bio-Sciences, Piscataway, NJ, USA). The membrane was blotted with specific antibodies. Antibodies against the following proteins were used: phosphorylated ATR (GeneTex, Inc., Irvine, CA, USA); cleaved PARP (Cell Signaling Technology, Danvers, MA, USA); ATR (Santa Crutz Biotechnology, Dallas, TX, USA); ATF4 (Santa Crutz Biotechnology); CHOP (Santa Crutz Biotechnology); phosphorylated H2Ax ( $\gamma \mathrm{H} 2 \mathrm{Ax}$; Merck-Millipore), p62 (Progen Biotechnik, Heidelberg, Germany) and Actin (Sigma-Aldrich, St. Louis, MO, USA).

Mouse experiments. All animal experiments were performed in accordance with EU directive 2010/63 (regional animal ethics committee of Gothenburg \#362014). The PDXs were obtained by injecting $2 \times 10^{5}$ cells mixed with equal volume of Matrigel (Corning, NY, USA) subcutaneously at the flank of immunocompromised, non-obese severe combined immune-deficient interleukin-2 chain receptor $\gamma$ knockout mice (NOG mice; Taconic, Ry, Denmark) as described previously. ${ }^{30}$
Tumors were measured with caliper at regular time points and tumor volume calculated using the formula: tumor volume $\left(\mathrm{mm}^{3}\right)=($ length $(\mathrm{mm})) \times(\text { width }(\mathrm{mm}))^{2} / 2$. B16F10-luciferase cells were transplanted by subcutaneous injection. Seven days after transplantation, mice were injected with $100 \mu \mathrm{l}$ of $30 \mathrm{mg} / \mathrm{ml}$ D-luciferin. Mice were sedated in an isofluran administrating chamber and then placed in an IVIS Lumina III XR machine (Perkin-Elmer, Norwalk, CT, USA).

Statistical analysis. Values are presented as mean \pm S.D. when data are combined. For statistical analyses, we used Graphpad Software, Inc. (La Jolla, CA, USA): multiple t-test or one-way ANOVA (with Sidak corrections) for tumor burden, and the log-rank test for survival. All mouse experiments contained 3-5 mice per group.

\section{Conflict of Interest}

EC is an employee, and JW was an employee, of Zenith Epigenetics Ltd when this project initiated. They contributed data in Supplementary Figure S1, read, edited and approved the submission but did not influence the design and interpretation of the other experiments. The remaining authors declare no conflict of interest.

Acknowledgements. We thank Sofia Nordstrand for assistance with animal experiments and Zenith Epigenetics for donating RVX2135. This work was supported by the Swedish Cancer Society, the Swedish Research Council, the Region Västra Götaland (Sahlgrenska University Hospital, Gothenburg), the Knut and Alice Wallenberg Foundation, the IngaBritt and Arne Lundberg Foundation, the Lion's Foundation West, the Familjen Erling Persson Foundation and BioCARE - a National Strategic Cancer Research Program at University of Gothenburg (to JAN), and from the Assar Gabrielsson Foundation, the W\&M Lundgren Foundation and Sahlgrenska Universitetssjukhusets stiftelser (Sahlgrenska University Hospital, Gothenburg) (to SVM, JB and BOE).

\section{Author contributions}

JAN conceived and supervised the study and wrote the paper. SVM, BOE, JB, MFL and JW performed experiments. ROB, US and LN recruited patients for PDX model generations. EC and LMN supervised aspects of the study. All authors read, edited and approved the manuscript before submission.

\section{Publisher's Note}

Springer Nature remains neutral with regard to jurisdictional claims in published maps and institutional affiliations.

1. Eggermont AM, Spatz A, Robert C. Cutaneous melanoma. Lancet 2014; 383: 816-827.

2. Davies H, Bignell GR, Cox C, Stephens P, Edkins S, Clegg $S$ et al. Mutations of the BRAF gene in human cancer. Nature 2002; 417: 949-954.

3. Sosman JA, Kim KB, Schuchter L, Gonzalez R, Pavlick AC, Weber JS et al. Survival in BRAF V600-mutant advanced melanoma treated with vemurafenib. N Engl J Med 2012; 366: 707-714.

4. Hauschild A, Grob JJ, Demidov LV, Jouary T, Gutzmer R, Millward M et al. Dabrafenib in BRAF-mutated metastatic melanoma: a multicentre, open-label, phase 3 randomised controlled trial. Lancet 2012; 380: 358-365.

5. Flaherty KT, Infante JR, Daud A, Gonzalez R, Kefford RF, Sosman J et al. Combined BRAF and MEK inhibition in melanoma with BRAF V600 mutations. N Engl J Med 2012; 367: 1694-1703.

6. Wagle N, Van Allen EM, Treacy DJ, Frederick DT, Cooper ZA, Taylor-Weiner A et al. MAP kinase pathway alterations in BRAF-mutant melanoma patients with acquired resistance to combined RAF/MEK inhibition. Cancer Discov 2014; 4: 61-68.

7. Wagle N, Emery C, Berger MF, Davis MJ, Sawyer A, Pochanard P et al. Dissecting therapeutic resistance to RAF inhibition in melanoma by tumor genomic profiling. J Clin Oncol 2011; 29: 3085-3096.

8. Sharma P, Allison JP. The future of immune checkpoint therapy. Science 2015; 348: 56-61.

9. Topalian SL, Sznol M, McDermott DF, Kluger HM, Carvajal RD, Sharfman WH et al. Survival, durable tumor remission, and long-term safety in patients with advanced melanoma receiving nivolumab. J Clin Oncol 2014; 32: 1020-1030.

10. Hodi FS, O'Day SJ, McDermott DF, Weber RW, Sosman JA, Haanen JB et al. Improved survival with ipilimumab in patients with metastatic melanoma. N Engl J Med 2010; 363: 711-723.

11. Shi J, Vakoc CR. The mechanisms behind the therapeutic activity of BET bromodomain inhibition. Mol Cell 2014; 54: 728-736. 
12. Belkina AC, Denis GV. BET domain co-regulators in obesity, inflammation and cancer. Nat Rev Cancer 2012; 12: 465-477.

13. Stratikopoulos EE, Dendy M, Szabolcs M, Khaykin AJ, Lefebvre C, Zhou MM et al. Kinase and BET inhibitors together clamp inhibition of PI3K signaling and overcome resistance to therapy. Cancer Cell 2015; 27: 837-851.

14. Siegel MB, Liu SQ, Davare MA, Spurgeon SE, Loriaux MM, Druker BJ et al. Small molecule inhibitor screen identifies synergistic activity of the bromodomain inhibitor CPI203 and bortezomib in drug resistant myeloma. Oncotarget 2015; 6: 18921-18932.

15. Bhadury J, Nilsson LM, Muralidharan SV, Green LC, Li Z, Gesner EM et al. BET and HDAC inhibitors induce similar genes and biological effects and synergize to kill in Myc-induced murine lymphoma. Proc Natl Acad Sci USA 2014; 111: E2721-E2730.

16. Ceribelli M, Kelly PN, Shaffer AL, Wright GW, Xiao W, Yang Y et al. Blockade of oncogenic IkappaB kinase activity in diffuse large B-cell lymphoma by bromodomain and extraterminal domain protein inhibitors. Proc Natl Acad Sci USA 2014; 111: 11365-11370.

17. Emadali A, Rousseaux S, Bruder-Costa J, Rome C, Duley S, Hamaidia S et al. Identification of a novel BET bromodomain inhibitor-sensitive, gene regulatory circuit that controls Rituximab response and tumour growth in aggressive lymphoid cancers. EMBO Mol Med 2013; 5: 1180-1195

18. Moros A, Rodriguez V, Saborit-Villarroya I, Montraveta A, Balsas P, Sandy P et al. Synergistic antitumor activity of lenalidomide with the BET bromodomain inhibitor CPI203 in bortezomib-resistant mantle cell lymphoma. Leukemia 2014; 28: 2049-2059.

19. Muralidharan SV, Bhadury J, Nilsson LM, Green LC, McLure KG, Nilsson JA. BET bromodomain inhibitors synergize with ATR inhibitors to induce DNA damage, apoptosis, senescence-associated secretory pathway and ER stress in Myc-induced lymphoma cells. Oncogene 2016; 35: 4689-4697.

20. Chai G, Li L, Zhou W, Wu L, Zhao Y, Wang D et al. HDAC inhibitors act with 5-aza-2'deoxycytidine to inhibit cell proliferation by suppressing removal of incorporated abases in lung cancer cells. PLOS ONE 2008; 3: e2445.

21. Lecona $E$, Fernandez-Capetillo $O$. Replication stress and cancer: it takes two to tango Exp Cell Res 2014; 329: 26-34.

22. Ferrao PT, Bukczynska EP, Johnstone RW, McArthur GA. Efficacy of CHK inhibitors as single agents in MYC-driven lymphoma cells. Oncogene 2012; 31: 1661-1672.

23. Murga M, Campaner S, Lopez-Contreras AJ, Toledo LI, Soria R, Montana MF et al. Exploiting oncogene-induced replicative stress for the selective killing of Myc-driven tumors. Nat Struct Mol Biol 2011; 18: 1331-1335.

24. Höglund A, Strömvall K, Li Y, Forshell LP, Nilsson JA. Chk2 deficiency in Myc overexpressing lymphoma cells elicits a synergistic lethal response in combination with PARP inhibition. Cell Cycle 2011; 10: 3598-3607.

25. Höglund A, Nilsson L, Muralidharan SV, Hasvold LA, Merta P, Rudelius M et al. Therapeutic implications for the induced levels of Chk1 in Myc-expressing cancer cells. Clin Cancer Res 2011; 17: 7067-7079.

26. Smith J, Larue L, Gillespie DA. Chk1 is essential for the development of murine epidermal melanocytes. Pigment Cell Melanoma Res 2013; 26: 580-585.
27. Gallagher SJ, Mijatov B, Gunatilake D, Tiffen JC, Gowrishankar K, Jin L et al. The epigenetic regulator I-BET151 induces BIM-dependent apoptosis and cell cycle arrest of human melanoma cells. J Invest Dermatol 2014; 134: 2795-2805.

28. Gallagher SJ, Mijatov B, Gunatilake D, Gowrishankar K, Tiffen J, James W et al. Control of $\mathrm{NF}-\mathrm{kB}$ activity in human melanoma by bromodomain and extra-terminal protein inhibitor l-BET151. Pigment Cell Melanoma Res 2014; 27: 1126-1137.

29. Bhadury J, Einarsdottir BO, Podraza A, Olofsson Bagge R, Stierner U, Ny L et al. Hypoxia-regulated gene expression explains differences between melanoma cell line-derived xenografts and patient-derived xenografts. Oncotarget 2016; 7 : 23801-23811.

30. Einarsdottir BO, Olofsson R, Bhadury J, Jespersen H, Mattsson J, Nilsson LM et al. Melanoma patient-derived xenografts accurately model the disease and develop fast enough to guide treatment decisions. Oncotarget 2014; 5: 9609-9618.

31. Prevo R, Fokas E, Reaper PM, Charlton PA, Pollard JR, McKenna WG et al. The novel ATR inhibitor VE-821 increases sensitivity of pancreatic cancer cells to radiation and chemotherapy. Cancer Biol Ther 2012; 13: 1072-1081.

32. Rogakou EP, Pilch DR, Orr AH, Ivanova VS, Bonner WM. DNA double-stranded breaks induce histone H2AX phosphorylation on serine 139. J Biol Chem 1998; 273: 5858-5868.

33. Soucek L, Whittield J, Martins CP, Finch AJ, Murphy DJ, Sodir NM et al. Modelling Myc inhibition as a cancer therapy. Nature 2008; 455: 679-683.

34. Hanahan D, Weinberg RA. Hallmarks of cancer: the next generation. Cell 2011; 144 646-674

35. Bryant HE, Schultz N, Thomas HD, Parker KM, Flower D, Lopez E et al. Specific killing of BRCA2-deficient tumours with inhibitors of poly(ADP-ribose) polymerase. Nature 2005; 434 913-917.

(c) Cell Death and Disease is an open-access journal published by Nature Publishing Group. This work is licensed under a Creative Commons Attribution 4.0 International License. The images or other third party material in this article are included in the article's Creative Commons license, unless indicated otherwise in the credit line; if the material is not included under the Creative Commons license, users will need to obtain permission from the license holder to reproduce the material. To view a copy of this license, visit http://creativecommons.org/licenses/by/4.0/

(C) The Author(s) 2017

Supplementary Information accompanies this paper on Cell Death and Disease website (http://www.nature.com/cddis) 